\title{
MEDICIÓN AUTOMÁTICA DEL NIVEL DE APRENDIZAJE EN NIÑOS DE 4 A 6 AÑOS DE EDAD APLICANDO TÉCNICAS DE PROCESAMIENTO DE SEÑALS
}

\section{AUTOMATIC MEASURE THE LEARNING LEVEL IN CHILDREN OF 4 TO 6 YEARS OLD APPLYING SIGNAL PROCESSING TECHNIQUES}

\author{
Ing. Ivette Stefany Flórez*, MSc. Luis Enrique Mendoza** \\ MSc.Viviana Pérez Ginna \\ Universidad de Pamplona \\ *** Grupo de Investigación en Ingeniería Biomédica, Semillero de investigación SIIBTEL \\ *** Grupo de investigación Comunicación Humana \\ E-mail: luis.mendoza@unipamplona.edu.co
}

\begin{abstract}
Resumen: Este artículo presenta la implementación de una herramienta que permita medir de manera automática y en tiempo real el grado de aprendizaje de niños entre edades de 4 a 6 años. El sistema contiene 10 sub-pruebas para la edad de 4 años, 11 subpruebas para la edad de 5 años y 13 sub-pruebas para la edad de 6 años. Para la implementación automática, se utilizó el procesamiento de señales de voz e imágenes. Las técnicas matemáticas usadas fueron: wavelet, morfología matemática, OTSU y algebra lineal. Se demostró que es posible medir el grado de aprendizaje de manera automática usando procesamiento digital de señales.
\end{abstract}

Palabras clave: Wavelet, morfología matemática, procesamiento de señales.

\begin{abstract}
This paper presents the implementation of a tool that allows the automatically measure and in real time the degree of learning of children between the ages of 4 and 6 years. The system contains 10 sub-tests for the age of 4 years, 11 sub-tests for the age of 5 years and 13 sub-tests for the age of 6 years. We used the processing of voice signals and image for automatic implementation. The mathematical techniques used were: wavelet, mathematical morphology, OTSU and linear algebra. Showed that it is possible, automatically measure the degree of learning using digital signal processing.
\end{abstract}

Keywords: Wavelet, mathematical morphology, signal processing.

\section{INTRODUCCIÓN}

El objetivo de la psicopedagogía es estudiar, prevenir y corregir dificultades y problemas de aprendizaje y ayudar a detectar tempranamente los niños con necesidades educativas especiales. Se encarga además, de diagnosticar, pronosticar, seguir y tratar al sujeto mediante procesos de enseñanza-aprendizaje [1]. La psicopedagogía se centra principalmente en el desenvolvimiento de las personas en el ámbito educativo. Por otro lado, la prueba PLON (Prueba de Lenguaje Oral Navarra) busca detectar precozmente niños en los cuales el desarrollo lingüístico no es el adecuado según su edad cronológica para poder actuar de forma compensatoria. Al realizar el análisis del nivel lingüístico de un niño se debe relacionar directamente con el nivel de desarrollo general de este. La prueba PLON consta de tres apartados principalmente (forma, contenido y uso), y estos a su vez, se dividen en otros sub-apartados [2]. En aquellos apartados se evalúa al infante en aspectos 
de morfología y sintaxis, los niveles de retraso o trastornos fonológicos, las variaciones en el léxico, la producción verbal de frases y el desarrollo cognitivo de los niños. Analiza el lenguaje infantil en aspectos de planificación, autorregulación, comprensión y adaptación, busca también identificar el potencial verbal del niño al comunicarse en situaciones habituales. La importancia que tiene este tipo de prueba es que puede ayudar a desarrollar la comprensión, concentración, memoria y atención. También logra identificar niños con dislexia, que son aquellos niños que presentan dificultad para la lectura, la escritura, y que tienen problemas de orientación espacial y temporal.

El aplicativo incentiva al niño a desarrollar su producción verbal y a mejorar la capacidad de comunicación e interacción con el mundo. Así mismo, logra motivar al niño a desarrollar las actividades escolares. Hace algunos años se ha utilizado el procesamiento digital de imágenes en ámbitos como la biología, medicina, telecomunicaciones y control de procesos industriales. El procesamiento brinda la posibilidad de manipular imágenes y señales captadas a través de un equipo electrónico.

El objetivo de realizar procesamiento es interpretar los datos para tomar decisiones automáticamente, ya que este consigue suplir la necesidad de que los sistemas funcionen en tiempo real. Algunas aplicaciones del procesamiento de imágenes es la detección de objetos, la medición de características geométricas, clasificación de objetos, mejoramiento de la calidad de imágenes, entre otros [3]. Así mismo, el procesamiento de voz ha permitido desarrollar diferentes aplicaciones como el dialogo entre el hombre y la máquina, síntesis a partir de texto, identificación y análisis de palabras y frases [3].

Con el uso de las diferentes técnicas de procesamiento de imágenes y de voz, se puede lograr la automatización de la prueba PLON, ya que el procesamiento de imágenes consigue identificar y seleccionar objetos de interés. Actualmente, existen distintos trabajos basados en la evaluación del nivel de aprendizaje, algunos de ellos son: SFC CASA, símbolos Fotográficos para la Comunicación: es un conjunto de programas informáticos creado con el fin de tratar personas afectadas neurológicamente y niños en proceso de aprendizaje [4]. PTAM, EXLER Y COFRE: se trata de una aplicación informática que sirve de apoyo para personas con trastornos del lenguaje oral y escrito [5]. Propuesta didáctica para el desarrollo de repertorios básicos de atención y memoria en niñas y niños con síndrome de Down integrados al aula regular [6]. Test Oral en Ingles del sistema escolar vocacional basado en red de campus [7].

Un sistema de síntesis del habla de bajo costo para la traducción de texto ASCII al lenguaje oral como una ayuda para problemas de visión [8]. Hacia el reconocimiento preciso de la fluidez oral en niños al leer [9]. Un sistema de reacción oral asistida inglesa - Un caso de estudio para calificar el nivel junior de inglés en Taiwán [10].

Generalmente, estas aplicaciones están orientadas a tratar personas adultas, ancianas y niños. El programa basado en la prueba PLON está pensando específicamente en niños en edades temprana. Además, no solo evalúa el lenguaje oral, sino que también mide la dificultad de aprendizaje que presenta o no el niño.

El aplicativo sirve de ayuda para el mejoramiento de las capacidades verbales y cognitivas del niño. Mide el grado de retraso o normalidad del lenguaje y aprendizaje que pueden presentar los niños en edades específicas, además permite ingresar resultados automáticamente a medida que aplica cada ejercicio, guarda los resultados en una base de datos para facilitar el análisis al especialista, y muestra graficas estadísticas.

\section{PRUEBA PLON}

La prueba PLON tiene como objetivo la detección temprana de niños con edades entre los 4 y 6 años con trastornos en el desarrollo del lenguaje, pues cuanto más pequeño es el niño, más fácil será la adquisición de nuevos aprendizajes.

En el test de valoración se utiliza procesamiento de voz, imágenes y video. La prueba está divida en tres apartados:

\subsection{Forma}

a) Fonología. Consta de un ejercicio basado en la identificación de fonemas.

b) Morfología y sintaxis. Se constituye de dos ejercicios basados en la evaluación de la producción verbal sugerida por imágenes, analizando el número de frases y de las palabras dichas en ellos. 


\subsection{Contenido}

a) Léxico. El objetivo en este sub-apartado es la identificación y clasificación de objetos dentro de una imagen.

b) Identificación de colores. El ejercicio de este sub-apartado busca conocer si el niño discrimina los colores rojo, amarillo, azul y verde.

c) Relaciones espaciales. Promueve al niño a realizar ejercicios que impliquen movimientos, como ubicar una ficha encima, debajo, delante o al lado de un objeto (un carro).

\subsection{Uso}

a) Interacción espontánea durante una actividad manipulativa. El ejercicio de esta sección busca analizar a través de la observación las conductas verbales producidas espontáneamente por el niño, mediante la realización de un rompecabezas.

\section{PROCESAMIENTO DE VOZ E IMÁGENES EN LA PRUEBA PLON}

A continuación, se describen las técnicas utilizadas durante la automatización de la prueba.

\subsection{Transformada Wavelet}

Esta técnica está especialmente indicada para señales no periódicas. Permite analizar señales en los dominios de tiempo y frecuencia, y de esta forma, poder observar los cambios espectrales de frecuencia a medida que transcurre el tiempo.

La transformada Wavelet viene dada por la ecuación (2):

$W T(a, \tau)=\frac{1}{\sqrt{a}} \int_{-\infty}^{\infty} s(t) \Psi^{*}\left(\frac{t-\tau}{a}\right) d t$

Donde:

$s(t)=$ Función a analizar.

$\Psi=$ Función Wavelet Madre.

$\tau=$ Traslaciones (desplazamiento temporal)

$\mathrm{a}=$ Dilatación o contracción (factor de escalamiento).

$t=$ Variable uni-dimensional continua.

La ecu (1), muestrea la señal $s(t)$ utilizando diferentes versiones de la función wavelet madre dilatados y trasladados, tomando punto a punto para realizar su respectivo análisis [11].

Algoritmo de conteo de palabras: una vez aplicado wavelet se procedió a tomar el promedio cada 100 datos, esto con el fin de tomar una envolvente de la señal de voz. Ya con la envolvente se aplicó el algoritmo de conteo de palabras, este algoritmo está descrito según:

$$
\text { If } x(i)>x(i-1) \& x(i)>x(i+1) p=p+1
$$

Donde $\mathrm{p}$ es un acumulador que contiene la cantidad de palabras que contiene la señal.

En el algoritmo se utilizó wavelet para eliminar el ruido de la señal y obtener la señal lo más parecida posible a la original de voz, con el fin de contar los picos y así obtener el número de palabras dichas por el niño.

\subsection{Método OTSU}

Este método se basa en la umbralización, la cual busca distinguir o separar los objetos y el fondo en una imagen binarizada, calculando el valor de cada pixel y comparándolo con un determinado valor de umbral.

El método OTSU permite determinar dicho umbral óptimo automáticamente, aplicando el criterio de máxima varianza entre clases [12].

Dado que una imagen en escala de grises, posee $N$ pixeles, en los cuales los niveles de gris se encuentran entre 1 y $L$.

El número de pixeles con nivel de gris $i$ se expresa como $f i$, la probabilidad de ocurrencia del nivel de gris $i$ en la imagen está dada por la ecuación (3):

$$
P i=\frac{f i}{N}
$$

En cuanto a la umbralización de dos niveles o binarización de una imagen, los pixeles se dividen en dos clases: $C 1$ con niveles de gris entre $[1, . ., t]$; y $C 2$ con niveles de gris entre $[t+1, \ldots ., L]$. En este caso, las distribuciones de probabilidad de los niveles de gris de ambas clases son:

$$
\begin{gathered}
C 1: \frac{P 1}{\omega 1(t)}, \cdots, \frac{P t}{\omega 1(t)} \\
C 2: \frac{P t+1}{\omega 2(t)}, \frac{P t+2}{\omega 2(t)}, \cdots, \frac{P L}{\omega 2(t)} \\
\text { Donde: } \omega 1(t)=\sum_{i=1}^{t} P i \text { y } \omega 2(t)=\sum_{i=t+1}^{L} P i \\
\mu 1=\sum_{i=1}^{t} \frac{i \cdot P i}{\omega 1(t)} \\
\mu 2=\sum_{i=t+1}^{L} \frac{i \cdot P i}{\omega 2(t)}
\end{gathered}
$$


Las ecuaciones (5) y (6) describen las medias para las clases $C 1$ y $C 2$.

Donde: $\omega 1+\omega 2=1$

$$
\omega 1 \cdot \mu 1+\omega 2 \cdot \mu 2=\mu T
$$

$\mu T$ es la intensidad Media Total de la imagen.

$$
\sigma_{B}^{2}=\omega 1(\mu 1-\mu T)^{2}+\omega 2(\mu 2-\mu T)^{2}
$$

La ecuación (8) describe como OTSU definió la varianza entre clases de una imagen umbralizada.

$$
t^{*}=\max \left\{\sigma_{B}^{2}(t)\right\} \quad 1 \leq t \leq L
$$

El método indica que para hallar el umbral óptimo $t^{*}$ en una imagen binarizada, se debe hallar la varianza $\sigma_{B}^{2}$ máxima entre clases.

\subsection{Morfología matemática}

La morfología matemática aplicada a imágenes, se basa en operaciones de teoría de conjuntos, hace referencia a la geometría y la forma de los objetos. Se utiliza en la interpretación de la estructura o forma de los objetos [13] y [14].

Las operaciones de morfología matemática usadas en este trabajo se centran en: filtrado por área, etiquetado, área. Para el filtrado por área se utiliza el comando bwareaopen de la siguiente manera.

$$
x=b \text { wareaopen }(B W, 6000)
$$

Donde BW es la imagen binarizada. La función elimina todos los objetos o componentes que tienen menos de 6000 pixeles.

\subsection{Algebra lineal}

En una figura geométrica el área se define como la superficie o espacio encerrado entre los límites de la figura.

Existen fórmulas establecidas que permiten calcular dicha área. Por ejemplo, el área de un triángulo está definida por la multiplicación de la base y la altura dividida entre dos.

Pero en imagenologia, el área se define como un escalar que especifica el número de pixeles en una región. Para el procesamiento de imágenes utilizadas en la prueba, se calcularon las propiedades de las regiones que contiene el objeto, como son los centroides de las regiones de la imagen, el área y el Boundingbox, que es un rectángulo que encierra una región.

\section{RESULTADOS}

A continuación, se presentan los resultados más relevantes del trabajo. La reproducción de frases, evalúa la producción verbal, donde a partir de la imagen (ver figura 1).

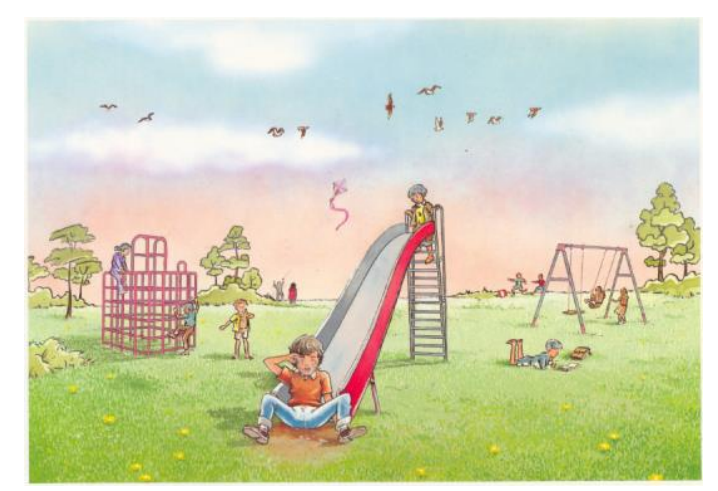

Fig. 1. Prueba a aplicar a niño de 4 años y 5 años

La figura 1, es tomada para aplicar la prueba a niño de 4 años y 5 años.

El software analiza el número de palabras emitidas por el niño. En la figura 1 se realizan pregunta como: ¿que observa?, describa la imagen. En este caso el algoritmo registra la señal de voz (ver figura 2), y cuanta el número de palabras o frases pronunciadas.

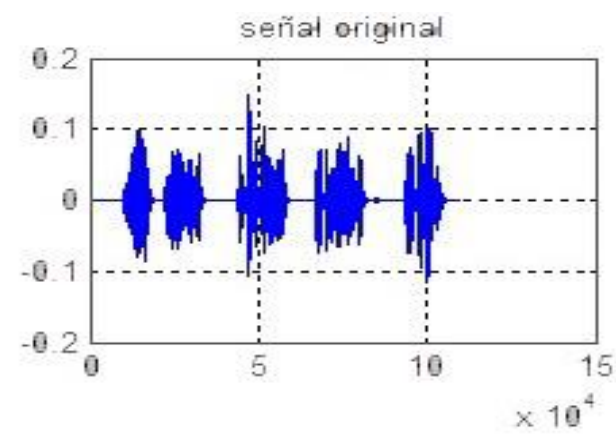

Fig. 2. Señal original.

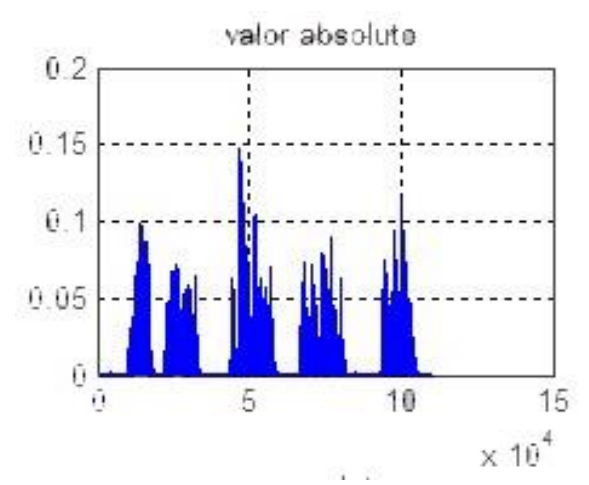

Fig. 3. Valor absoluto. 


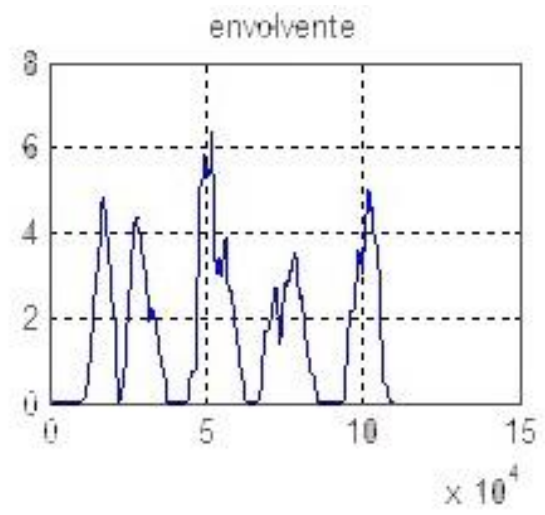

Fig. 4. Envolvente de la señal.

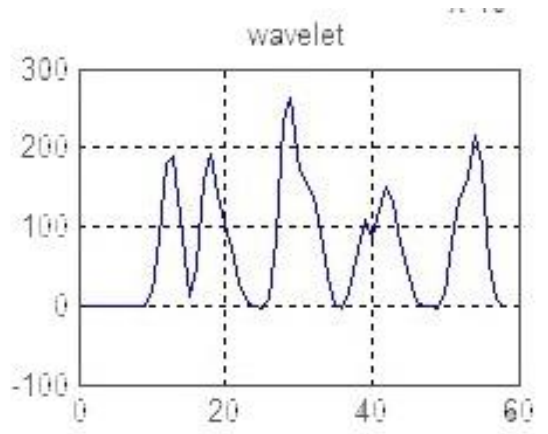

Fig. 5. Trasformada Wavelet de la señal.

Para contar el número de palabras de cada señal se realizó un proceso de análisis wavelet, como se observa en la figura 5.

En una segunda prueba, se reproducen una serie de frases como:

Frase 1. "El gato cazó un ratón en el patio".

Frase 2. "La maestra tiene cuentos para los niños" Frase 3."Mi amigo tiene un canario amarillo que canta mucho",

Frase 4."Tarzán y la mona Chita corrían mucho porque les perseguía un león"

Las frases 1 y 2 son usadas para evaluar a los niños de 4 años. $Y$ las frases 3 y 4 son usadas para evaluar a los niños de 5 años.

El software aplica el conteo de palabras que el niño pronuncio en cada frase. El algoritmo registra la señal de voz (ver figura 6), y cuanta el número de palabras o frases pronunciadas.

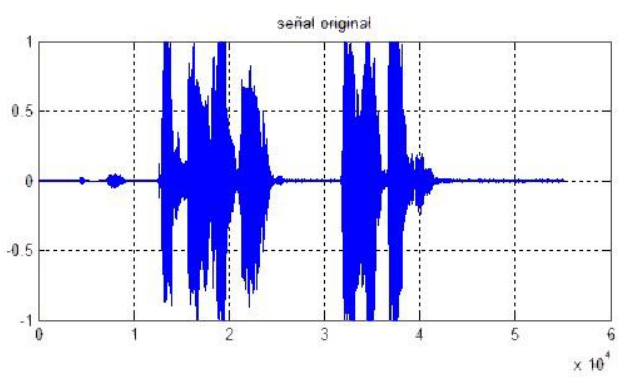

Fig. 6. Señal original.

Se aplica el valor absoluto a la señal, sumado de un promedio cada 600 puntos con el fin de simular una envolvente de la señal (ver figura 7). Aquí se aplica el algoritmo de conteo de picos.

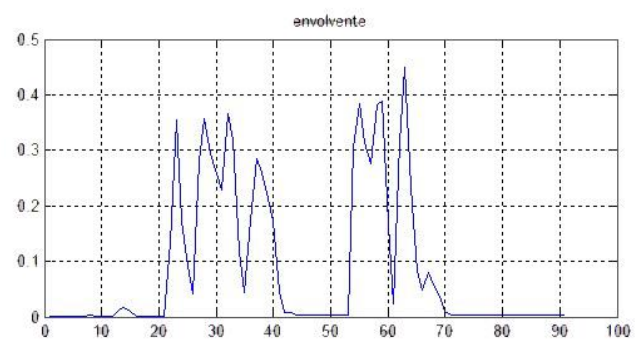

Fig. 7. Envolvente de la señal.

Por otro lado, en una tercera prueba, se trabaja la destreza del niño para la selección de objetos dentro de una imagen cualquiera.

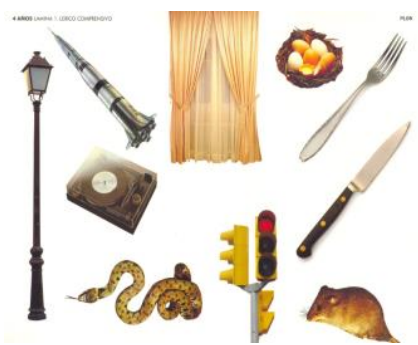

Fig. 8. Muestra 1 de objeto a seleccionar.

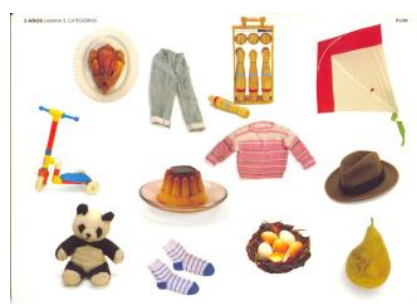

Fig. 9. Muestra 2 de objeto a seleccionar. 


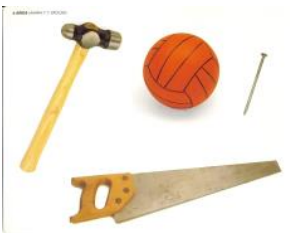

Fig. 10. Muestra 3.

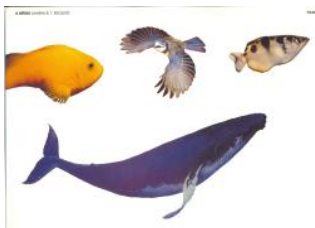

Fig. 11. Muestra 4.

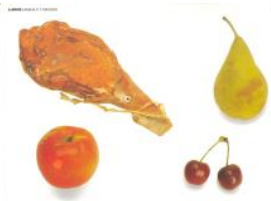

Fig. 12. Muestra 5.

La figura 8, es tomada para aplicar la prueba a niño de 4 años, la figura 9 es utilizada para niños de 5 años y la figura 10, 11 y 12 es utilizada para niños de 6 años. Para que el sistema pueda reconocer que objeto esta seleccionando el niño, se procedió a condicionar la imagen en estado binario, como se observa en las figuras 13, 14, 15, 16 y 17.

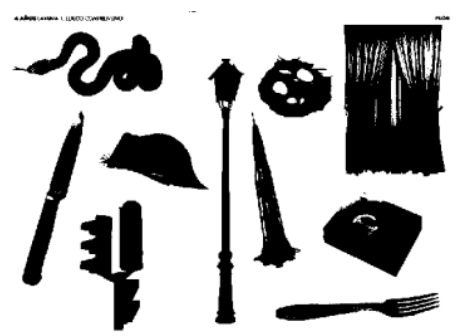

Fig. 13. Muestra 1 en binario.

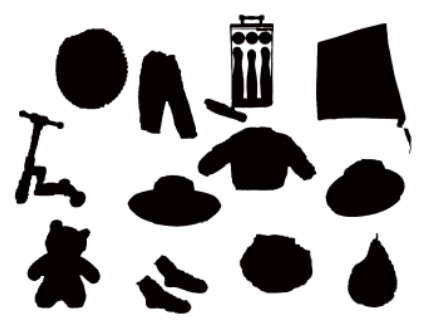

Fig. 14. Muestra 2 en binario.

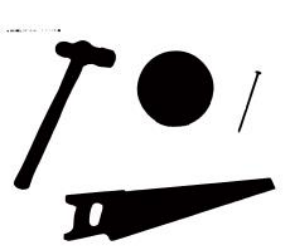

Fig. 15. Muestra 3.

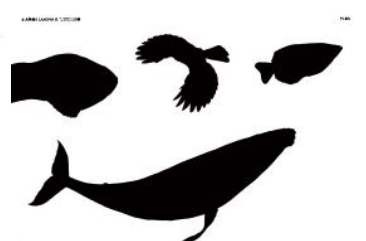

Fig. 16. Muestra 4.

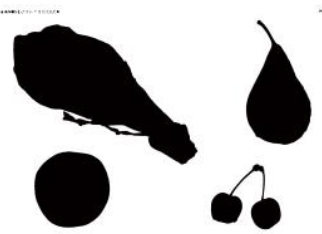

Fig. 17. Muestra 5 en binario.

En la figura 13 a la 17 , se observan unas imágenes binarias donde los objetos son negro y el sistema necesariamente debe contener los objetos a reconocer en color blanco, por esto se hace una transformación de color como se observa en las figuras $18,19,20,21$ y 22.

Adicionalmente para evitar punto negro dentro de los objetos se procedió a utilizar la función "imclose", que funciona como un cierre morfológico mediante una dilatación seguida de una erosión. Finalmente se obtuvieron las propiedades de los objetos tales como: área y centroide, que son dos valores muy representativos de los objetos de la imagen.

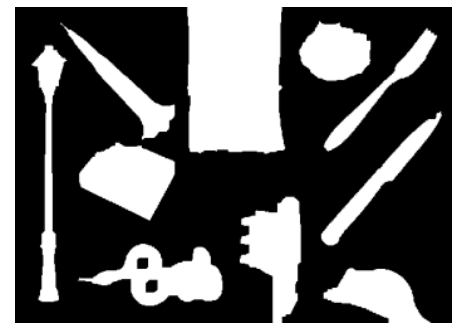

Fig. 18. Muestra 1 con función imclose.

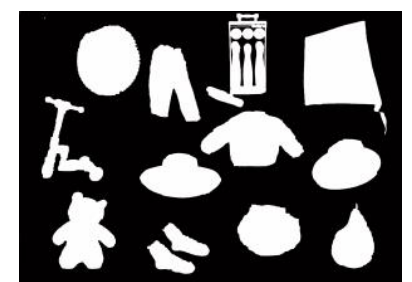

Fig. 19. Muestra 1 con función imclose.
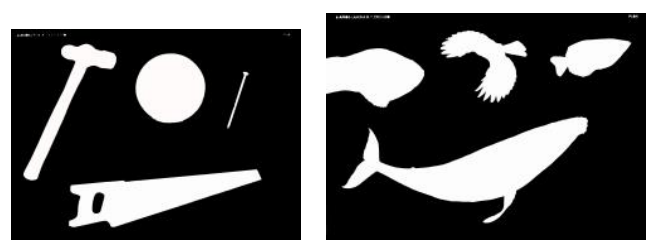

Fig. 20. Muestra 3.

Fig. 21. Muestra 4.

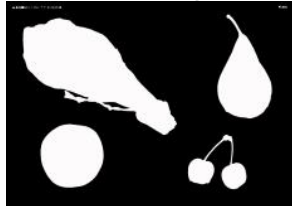

Fig. 22. Muestra 5 con función imclose. 
En este tipo de pruebas se pretende evaluar el vocabulario comprensivo del niño. Para esta prueba existen diferentes figuras (ver figuras de la 8 a la 12), que son utilizadas, el sistema implementado aleatoriamente ejecuta la acción de seleccionar 6 objetos de la figura, es importante resaltar que los 6 objetos no siempre son los mismo y que la prueba garantiza que los objetos cambien de lugar, como se observa en las figuras 23 a la 32 .

\section{4 años}

Versión 1 Versión 2

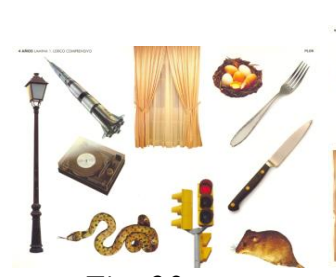

Fig. 23

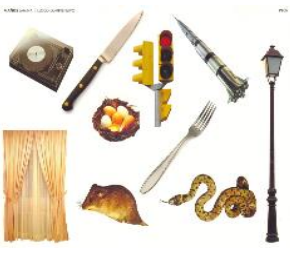

Fig. 24
5 años

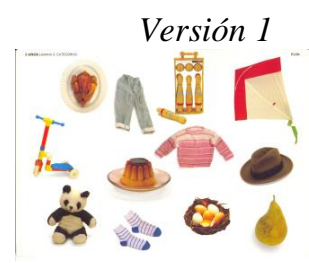

Fig. 25

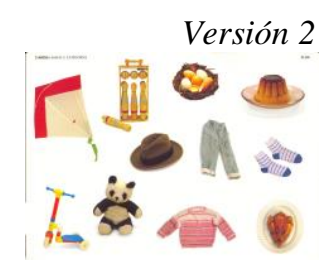

Fig. 26
6 años
Versión 1

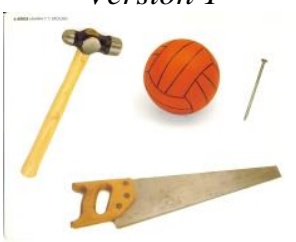

Fig. 27

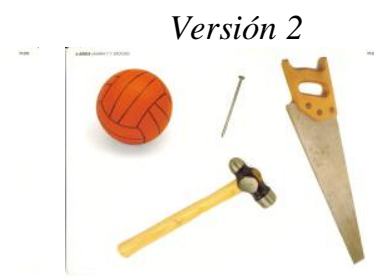

Fig. 28
Versión 1

Versión 2

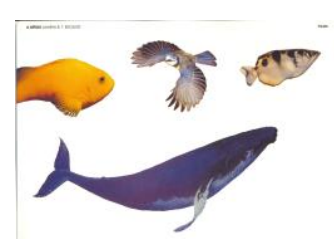

Fig. 29

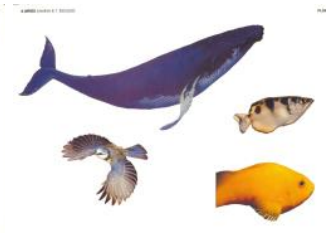

Fig. 30
Versión 1

Versión 2

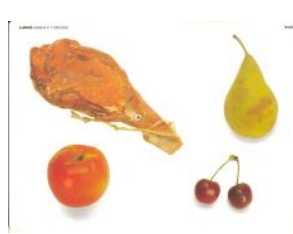

Fig. 31

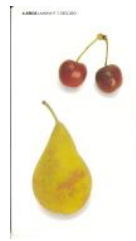

Fig. 32
Finalmente se muestra la fase inicial de la interfaz final que se lleva a cabo en este momento (ver figura 33), es importante resaltar que el trabajo esta comenzado las pruebas con sujetos y que se tiene pronosticado realizar mas de 50 pruebas con niños.

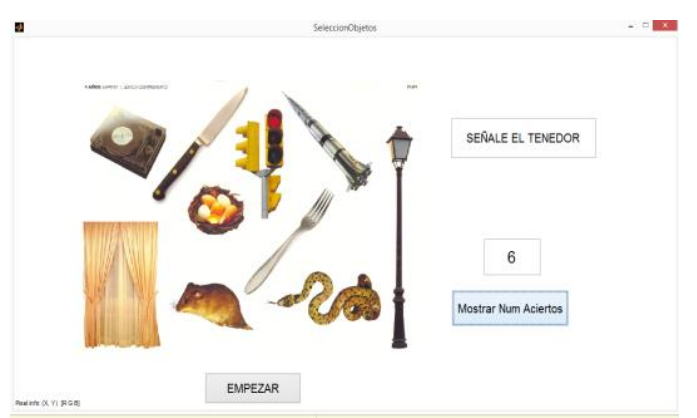

Fig. 33.

\section{CONCLUSIONES}

El automatizar la prueba PLON permitió realizar de manera mas rápida y con mayor eficiencia las pruebas y tener resultados en tiempo real sobre las cualidades de los niños entre 4-6 años de edad en cuanto a a el aprendizaje se refiere por otro lado este sistema permite ir incluyendo pruebas con el fin de lograr tener en un futuro tener un sistema robusto y que pueda analizar desde procesamiento de voz e imágenes hasta usar la visión artificial para detectar comportamiento y ejecución de acciones como: colocar arriba de, llevar a la izquierda, y otras acciones importantes. Finalmente, el sistema tiene un rendimiento en tiempo en procesamiento de voz de: en segundo, en selección de imágenes es de un segundo. Esto indica que es posible presentar los resultados en todo el examen en menos de un segundo una vez termine el niño de realizar la prueba.

\section{REFERENCIAS}

[1] Funciones del Psicopedagogo,» [En línea]. Available:

[2]. http://www.psicopedagogia.com/articulos/?ar ticulo=335. [Último acceso: 25 agosto 2015]. 
[2] G. Aguinaja Ayerra, M. L. Armentia Lopez, A. Fraile Blazquez, P. Olangua Baquedano y N. Uriz Bidegain, PLON - Prueba de Lenjuaje Oral Navarra, Gobierno de Navarra - Departamento de Educación y Cultura. .

[3] Procesamiento de imágenes y voz,» [En línea]. Available: http://fraba.galeon.com/imagenvoz.htm. [Último acceso: 30 Agosto 2015].

[4] MIRELO,» [En línea]. Available: http://www.mirelo.net/fiche.php?id=8\&PHP SESSID=687bc6797c8feaf $1628 b f e 12955 f 31 \mathrm{f}$ 8.\%20[\%C3\%9Altimo\%20acceso:\%2005\%2 009\%202015. [Último acceso: 3 Septiembre 2015].

[5] C. B. Ortí, «Los programas de la Escola de patología,» [En línea]. Available: http://www.uv.es/bellochc/logopedia/epl.pdf . [Último acceso: 4 Septiembre 2015].

[6] N. Niño y D. A. Ramírez Salazar. [En línea]. Available: http://aprendeenlinea.udea.edu.co/revistas/in dex.php/revistaeyp/article/view/6031. [Último acceso: 28 Agosto 2015].

[7] H. Xingyun, «IEEE Xplore,» [En línea]. Available: http://ieeexplore.ieee.org/search/s earchresult.jsp?queryText=Navarra\%20oral $\%$ 20language $\%$ 20test\&newsearch=true.

[8] J. Ramirez, «IEEE Xplore,» [En línea]. Available: http://ieeexplore.ieee.org/search/searchresult. jsp?queryText=Navarra\%20oral\%20languag e \%20test\&newsearch=true. [Último acceso: 23 Septiembre 2015].

[9] J. Cheng, «IEEE Xplore,» [En línea]. Available: http://ieeexplore.ieee.org/search/searchresult. jsp?queryText=Navarra\%20oral\%20languag e\%20test\&newsearch=true. [Último acceso: 24 Septiembre 2015].
[10] H.-M. L. Chien Hsien Huang, «IEEE Xplore,» [En línea]. Available: http://ieeexplore.ieee.org/search/searchresult. jsp?queryText=Navarra\%20oral\%20languag e\%20test\&newsearch=true. [Último acceso: 24 Septiembre 2015].

[11] P. Turmero, «monografias.com,» [En línea]. Available:

http://www.monografias.com/trabajos105/wa velets-concepto-y-aplicaciones-analisissenales/wavelets-concepto-y-aplicacionesanalisis-senales.shtml. [Último acceso: 1 septimbre 2015].

[12] Método de Ostu (Segmentación por umbralización),» [En línea]. Available: www.ilopez.es/proyectos/matematicas/Otsu. pptx. [Último acceso: 25 Septiembre 2015.

[13] A. Rojas, S. Diaz y A. Betancourt, «Sistema de detección y reconocimiento de falla en un tipo de tarjeta electrónica de celulares,» 2004. [En línea]. Available: http://revistas.udistrital.edu.co/ojs/index.php/ reving/rt/printerFriendly/1886/2462. [Último acceso: 2 Septiembre 2015].

[14] E. de la Fuente López y F. M. Trespaderne, «Morfología Matemática - vision artificial industrial,» [En línea]. Available: www.librovision.eii.uva.es/ppt/5_Morfologia .pptx. [Último acceso: 4 Septiembre 2015].

[16] Procesamiento de imágenes y voz,» [En línea].

Available: http://fraba.galeon.com/imagenvoz.htm. [Último acceso: 30 Agosto 2015]. 